\title{
Les élections dans les opérations internationales de pacification : un instrument de réconciliation? Une réflexion sur la Bosnie
}

Maria del Mar Bermudez et Juan Carlos Guerrero

\section{(2) OpenEdition \\ Journals}

Édition électronique

URL : http://journals.openedition.org/conflits/492

DOI : $10.4000 /$ conflits.492

ISSN : $1777-5345$

Éditeur :

CCLS - Centre d'études sur les conflits lilberté et sécurité, L'Harmattan

Édition imprimée

Date de publication : 1 septembre 2000

ISBN : 2-7475-0572-3

ISSN : 1157-996X

\section{Référence électronique}

Maria del Mar Bermudez et Juan Carlos Guerrero, «Les élections dans les opérations internationales de pacification : un instrument de réconciliation? Une réflexion sur la Bosnie », Cultures \& Conflits [En ligne], 40 | hiver 2000, mis en ligne le 28 septembre 2006, consulté le 30 mars 2021. URL : http:// journals.openedition.org/conflits/492 ; DOI : https://doi.org/10.4000/conflits.492

Ce document a été généré automatiquement le 30 mars 2021.

Creative Commons License 


\title{
Les élections dans les opérations internationales de pacification : un instrument de réconciliation? Une réflexion sur la Bosnie
}

\author{
Maria del Mar Bermudez et Juan Carlos Guerrero
}

1 Les élections dans les opérations internationales de pacification: un instrument de réconciliation? Une réflexion sur la Bosnie Juan Carlos GUERRERO, María del Mar BERMUDEZ

2 Exercice de participation politique par excellence, les élections apparaissent progressivement comme un volet incontournable des opérations internationales de pacification. Bien entendu, ce n'est pas un phénomène complètement nouveau. La tenue d'élections fut, par exemple, imposée par les alliés au Japon à la fin de la deuxième guerre mondiale, de même qu'elle constitua une condition préalable à la décolonisation espagnole du Sahara occidental au cours des années soixante-dix. Le phénomène a pourtant tendance à se banaliser depuis la fin de la guerre froide. Lors de l'intervention de la communauté internationale dans la définition de sorties négociées de conflits intra-étatiques, les scrutins apparaissent en effet comme une forme de participation politique fortement valorisée, voire idéalisée. Dans la perspective occidentale, le vote est un élément constitutif de la démocratie: "expression périodique des opinions et des préférences politiques des citoyens $»^{1}$, il est, selon un avis désormais unanime, une condition sine qua non de légitimité de l'ordre politique. Sans élections, il n'y a donc pas d'État démocratique. Ainsi, qu'il s'agisse du Cambodge, du Liberia, de Haïti, du Salvador, du Guatemala, de la Palestine, de la Bosnie, l'exercice électoral se présente comme un instrument fondamental pour la construction ou la reconstruction de la citoyenneté et des institutions démocratiques des sociétés défaites par la guerre. Activité fondamentale de la vie politique démocratique institutionnalisée et routinisée, les élections sont également perçues comme l'occasion d'un «apprentissage du politique»: elles inculquent au sein d'un groupe social 
l'affrontement politique sans violence, l'affrontement verbal ${ }^{2}$. Lors du double défi du rétablissement de la paix (peace building) et de la reconstruction de l'État effondré (state building), la communauté internationale peut ainsi être amenée à considérer les élections comme une pratique qui rend possible le dépassement des affrontements violents. Au-delà des raisons normatives qui amènent à cette valorisation fort fréquente des élections en tant qu'instrument de la démarche pacificatrice, des questions d'ordre pratique se posent : dans quelle mesure la tenue d'élections rend-elle possible le passage de la guerre à la paix, tout en permettant la réconciliation au sein d'une communauté politique déchirée? Est-ce que l'affrontement verbal propre aux élections est forcément non-violent? Est-ce que les élections «inculquent» la valorisation de l'affrontement non-violent, ou en sont simplement exemplaires ou illustratives? On peut, en effet, concevoir des scénarios où la tenue d'élections, loin de pacifier les conflits, les aggrave. L'ambiguïté de la technique électorale sera étudiée en observant le déroulement des élections en Bosnie-Herzégovine après la signature de l'accord de Dayton. Ce cas est intéressant non seulement par la complexité et l'intensité du conflit, mais aussi par le rôle attribué aux élections dans le processus de pacification du pays. En effet, le vote y a été fortement valorisé comme instrument pacificateur. Même avant d'aboutir à l'établissement du cessez-le-feu (octobre 1995) et à la signature de l'accord de paix de Dayton (novembre 1995), de nombreux plans de paix dessinés par la communauté internationale avaient déjà envisagé l'organisation d'élections comme un préalable à tout passage solide à la paix. Dans les accords de Genève et de New York (septembre 1995), par exemple, la tenue d'élections n'était pas simplement un point incontournable de l'agenda des négociations, mais une condition sine qua non à tout accord de paix possible entre les parties. Si bien que l'accord de Dayton (novembre 1995) comporte une annexe (l'Annexe III : accord sur les élections) consacrée à l'organisation d'élections « libres et démocratiques » sous la tutelle de la communauté internationale. Les élections y sont envisagées comme un moyen, un instrument, pour " établir les bases d'un gouvernement représentatif » et pour assurer «la réalisation progressive des objectifs démocratiques en Bosnie $»^{3}$. L'accord de Dayton semble ainsi attribuer à l'exercice électoral un rôle fondamental : celui de l'institutionnalisation du politique (dans le sens de la création d'un champ social dominé par des conflits d'intérêts régulés par un pouvoir prétendant à la monopolisation de la coercition légitime), voire de l'institutionnalisation de la démocratie (dans le sens de l'établissement d'un régime politique représentatif). Dans ce sens, les élections prennent part au processus de réconciliation politique (reconnaissance de l'ancien adversaire en tant qu'acteur politique et non plus en tant qu'ennemi ou simple criminel). Or, la reconnaissance réciproque n'accomplit pas tout l'exercice de pacification. Le dépassement définitif de l'inimitié repose par ailleurs, à défaut d'un oubli peu probable à court terme, sur un processus moral de remords et de pardon. En tant qu'occasion d'un affrontement verbal non-violent, les élections pourraient, dans certaines conditions, contribuer au processus de réconciliation morale. Il apparaît alors légitime de s'interroger sur les implications de l'organisation d'élections dans le processus de réconciliation politique et morale des sociétés placées à l'articulation incertaine de la guerre et de la paix. Tel est le but de cet article ${ }^{4}$. De la signature de l'accord de Dayton aux premières élections en Bosnie : une esquisse de réconciliation politique. Issu de "la diplomatie de la carotte et du bâton ", l'accord de Dayton est souvent perçu comme une espèce de pax americana en Bosnie. Certes, à la fois en promettant des récompenses en cas d'accord et en proférant des menaces en cas de 
faillite des négociations, les États-Unis devinrent un médiateur manipulateur et musclé capable d'imposer des solutions et des formules de paix aux belligérants. Pourtant, la puissance de la diplomatie américaine lors du déroulement des négociations ne fut pas absolue. Si la croisade diplomatique de Washington modifia la vulnérabilité des parties face à l'échec des négociations, les acteurs de la guerre disposèrent également d'une ample marge de manœuvre. Toutes les ambiguïtés et les généralités de l'accord de Dayton en témoignent, notamment en ce qui concerne la définition de la nature et du cadre institutionnel du nouvel État bosnien ${ }^{5}$. Ne pouvant obtenir le consentement des belligérants sur un véritable cadre de cohabitation intercommunautaire, l'accord de Dayton représente un décor pauvre pour n'importe quel processus de pacification. En effet, l'accord ne constitue pas le moment de la réconciliation. Il représente simplement l'armistice, produit de la reconnaissance de la fatigue et de l'impasse de la guerre. Ce constat est sans doute révélateur des motivations des belligérants pour s'asseoir à la table de négociations avant la signature de l'accord. Il ne s'agissait pas véritablement de la recherche d'une paix profonde : les combattants participèrent au processus de négociation parce qu'il représentait le moment de la définition légitime des résultats de la guerre sur le terrain (la délimitation de frontières intercommunautaires reconnues par la communauté internationale). Il fallait donc être présents dans ce "partage des dépouilles ». Malgré cela, la signature de l'accord a eu des implications importantes pour le processus de réconciliation. Tout d'abord, il implique l'acceptation en principe de la fin de la guerre : l'affrontement armé prend fin, même si des conflits pouvaient soudainement éclater à nouveau entre les différentes communautés. Ensuite, en incorporant l'annexe sur les élections, l'accord déplace la confrontation du champ de bataille à l'arène politique : c'est sans doute un cadre plus favorable pour le processus de réconciliation. Enfin - et c'est bien ici que repose la véritable esquisse de la réconciliation - l'armistice amène à la reconnaissance de l'ennemi. L'ancien ennemi devient dorénavant un interlocuteur reconnu, un représentant d'une fraction du peuple. Il s'agit bien d'une reconnaissance réciproque mais, dans un premier temps, celle-ci n'a aucune légitimité démocratique. Ceux qui se reconnaissent ne sont que des chefs militaires dépourvus du pouvoir et de la légitimité qu'une élection populaire confère. Bien entendu, cela n'exclut nullement qu'ils puissent avoir du pouvoir et de la légitimité d'une autre source, par exemple de type charismatique. D'où l'importance des premières élections pour le processus de réconciliation politique. Bien qu'elles institutionnalisent une structure politique fragile et précaire, elles permettent au moins la désignation d'interlocuteurs et d'acteurs politiques à légitimité complète. Certes, le risque de légitimation démocratique des chefs militaires est fort présent lors de l'organisation des élections. Pourtant, la capacité des militaires à représenter le groupe dont ils se réclament peut également être mise en cause dans la joute électorale. En effet, des querelles de légitimité internes aux camps en présence confrontant des acteurs civils et des (para-) militaires y sont toujours possibles. Ainsi, les armes, même victorieuses, n'offrent plus aux militaires un monopole de représentation de la communauté au nom de laquelle ils ont combattu. En recoupant à angle droit le clivage du conflit, cette nouvelle opposition pourrait contribuer au processus de pacification politique. L'hypothèse est déjà formulée dans un des théorèmes classiques de la sociologie politique : la conflictualité globale d'une société diminue quand les enjeux de conflit en son sein ne se recoupent pas, autrement dit, quand ils sont cross-cutting. Par ailleurs, quelle que soit la véritable légitimité des acteurs civils élus, ils sont « utiles » au processus de pacification par le simple fait de ne 
pas être les mêmes que les acteurs de la guerre. Sans ces acteurs, en tout cas, la continuation de l'exercice de pacification s'avère irréalisable. Afin d'éviter la légitimation des anciens acteurs de la guerre en tant qu'acteurs politiques, l'Annexe III de l'accord de Dayton incorpora une série de dispositifs constituant un cadre régulateur de l'exercice électoral. En effet, l'accord ne se limita pas à énoncer les conditions d'élections "libres et démocratiques $»^{6}$. Au-delà, la communauté internationale fut tenue pour principale garante et responsable non seulement de la préparation, mais également $\mathrm{du}$ déroulement $\mathrm{du}$ processus électoral ${ }^{7}$. Par l'intermédiaire de l'Organisation pour la sécurité et la coopération européenne (OSCE), la communauté internationale s'assigna ainsi de manière transitoire, mais indéfinie dans le temps, un ensemble de tâches généralement accomplies par l'État dans des conditions normales. Selon Dayton, l'OSCE devait évaluer et patronner la présence des conditions minimales favorables au développement des élections; établir le programme des scrutins, en tenant compte des limites temporelles fixées à Dayton ${ }^{8}$; édicter les règles électorales et en vérifier le respect, en ayant même la faculté de sanctionner toute personne ou groupe désobéissant aux règles ; accréditer les observateurs internationaux et garantir les conditions de l'accomplissement de leur mission; rendre publics et certifier les résultats des scrutins. Bien évidemment, c'était un ensemble de responsabilités qui dépassait la simple observation du processus électoral et qui exigeait la mobilisation d'une force militaire internationale ${ }^{9}$. Cette force devait assurer non seulement le maintien de l'arrêt des hostilités, mais aussi la liberté de déplacement aux urnes et l'exercice du vote libre de toutes représailles ou pression. Par ailleurs, afin d'éviter la légitimation du «nettoyage ethnique » conduit pendant la guerre, le texte de Dayton fixa certains critères d'inscription des votants pour les premières élections nationales ${ }^{10}$. En Bosnie, tout citoyen bosnien d'au moins 18 ans, enregistré dans le recensement de 1991 pouvait voter, éventuellement par correspondance, dans la municipalité où il fut recensé à cette époque. Pour les fonctionnaires de l'OSCE, le droit de libre circulation des réfugiés le jour du vote devait être garanti, car la remise en question du «nettoyage ethnique " était une condition nécessaire à la réintégration du pays ${ }^{11}$. Les élections étaient donc non seulement conçues comme une pratique de participation politique, mais aussi comme un moyen pour inciter les réfugiés à exercer leur droit à reprendre possession de leur ancien lieu d'habitation, permettant ainsi le renversement des résultats de la guerre. Bien sûr, en stimulant la mémoire, la réinstallation dans l'ancien lieu d'habitation rend difficile le processus de réconciliation. Cela semble évident du coté des victimes de la guerre: les séquelles de la guerre demeurant vives dans la mémoire collective, plusieurs déplacés préfèrent alors rester dans leur nouveau lieu d'habitation, même si cela signifie consentir à la partition communautaire du pays et légitimer l'armistice intercommunautaire. Or, en dépit de toutes les dispositions mentionnées ci-dessus, les résultats des premières élections générales en Bosnie légitimèrent en tant qu'acteurs politiques les anciens acteurs de la guerre. Tel était le cas de Radovan Karadzic - inculpé pour crimes de guerre par le Tribunal pénal international pour la Yougoslavie - qui jusqu'en 1998 poursuit officieusement ses activités au sein du SDS serbe. De nombreux anciens chefs militaires participèrent au processus politique, parfois en agissant de façon clandestine mais de toute façon avec une certaine complicité de la part de la population locale ${ }^{12}$. On peut, à cet égard, trouver décevants les résultats des élections. Il ne faut pas oublier, cependant, que l'acceptation du compromis électoral de la part des belligérants ne releva pas d'une imposition pure et simple de la communauté internationale. Tout en envisageant de 
s'ériger en acteurs du jeu politique local, les acteurs de la guerre requéraient aussi une pratique de participation politique à fonction de légitimation. Dans la perspective des anciens combattants, le compromis formel de tenue d'élections ne représentait en fait qu'une concession mineure dans le processus de négociation. Les vrais enjeux étaient les conditions effectives du scrutin. Ces conditions établissaient la marge de manipulation potentielle du jeu électoral. Si l'accord de Dayton établit de nombreuses normes visant à créer les conditions d'un scrutin libre et démocratique, il n'en est pas moins vrai qu'il contient des ambiguïtés permettant aux anciens combattants la manipulation de la joute électorale. En même temps que Dayton reconnaît le droit de voter au lieu de résidence au moment du recensement de 1991, il consacre le droit pour les réfugiés et les déplacés de s'inscrire et voter dans la municipalité « où ils habitent depuis la fin de la guerre ou dans la municipalité où ils prévoient de vivre à l'avenir ». L'accord devient ainsi contradictoire. Il envisage d'abord le renversement des résultats de la guerre (reconnaissance du droit de retour des populations déplacées et du droit de voter dans la municipalité d'origine), mais laisse pourtant ouverte la possibilité de légitimer les déplacements forcés de population liés à la guerre (reconnaissance des lignes de division intercommunautaires établies par le nettoyage ethnique et du droit de voter dans une municipalité différente de celle d'origine). Il ouvre même la possibilité de nouveaux déplacements de population, car il reconnaît le droit à voter dans la municipalité où les déplacés envisagent d'habiter à l'avenir. Ainsi, les anciens combattants ont pu interpréter et détourner à leur avantage les directives de l'OSCE, notamment au moment de la constitution des listes électorales. La règle qui permettait aux réfugiés et aux déplacés de voter dans un lieu différent de celui où ils habitaient avant la guerre fut utilisée afin de confirmer et continuer le nettoyage ethnique de la guerre. En utilisant différents moyens de pression, les partis nationalistes des anciens combattants poussèrent la population à voter dans certaines villes où ils voulaient consolider leur pouvoir. Les Serbes, par exemple, recoururent au chantage, en utilisant l'aide humanitaire et l'assignation des foyers. Dans des villes à majorité serbe, les déplacés serbes devaient, pour obtenir des vêtements et de la nourriture, renoncer à voter dans leur village d'origine. Par ailleurs, les autorités serbes de Bosnie poussèrent la moitié des 240.000 réfugiés serbes en Serbie à s'inscrire dans des villes serbes où ils n'avaient pas l'intention de s'installer. Tout compte fait, pour obtenir une victoire électorale serbe dans une ville prise aux Bosniaques pendant la guerre, il suffisait de faire enregistrer assez de Serbes pour contrebalancer le pouvoir électoral des Bosniaques qui, enregistrés en 1991, pouvaient aller voter. De cette manière, Srebrenica, une ville de 16.000 habitants, dont 10.000 Serbes, en 1991, avait 24.394 Serbes inscrits sur les listes pour les élections municipales en $1996^{13}$. Certes, cette situation irrégulière obligea la communauté internationale à reporter les élections municipales de septembre 1996 à septembre 1997. Néanmoins, la pratique du vote conduit ou obligé ne disparut pas complètement lors de la tenue des premières élections générales en 1996, d'autant que les réglementations sur le retour des réfugiés ou des déplacés internes à leurs villes d'origine n'attribuèrent pas suffisamment de pouvoir de contrôle à la communauté internationale. En fait, le contrôle de la mise en œuvre des dispositions sur les élections tombait souvent dans les mains des acteurs locaux. Ainsi, malgré la disposition de Dayton qui permettait aux populations de voter dans leurs villes d'origine, si elles envisageaient d'y retourner, les premières élections générales en Bosnie ne servirent qu'à légitimer le nettoyage ethnique conduit pendant la guerre. En effet, le taux élevé de participation électorale n'était pas révélateur d'un 
libre mouvement interne de la population : d'une part, $22 \%$ des électeurs inscrits, soit 641.000 personnes ${ }^{14}$, étaient des réfugiés hors du pays qui exercèrent leur droit de vote à l'étranger, sans retourner en Bosnie ; d'autre part, seuls 24.000 électeurs à l'intérieur du pays, soit moins de $1 \%$ de l'électorat, franchirent la ligne de démarcation entre les deux entités pour aller voter dans leur lieu de résidence d'avant-guerre ${ }^{15}$. Étant donné la quantité de forces militaires et d'observateurs internationaux déployée pour surveiller le processus électoral, les mouvements de population entre les deux entités politiques du pays apparaissent encore plus insignifiants. Même dotée de la faculté aussi bien d'intervenir pour éviter les incidents et les affrontements que de détenir temporairement les fauteurs de troubles, l'IFOR ne put garantir le libre déplacement de la population locale. De la signature de l'accord de Dayton aux premières élections nationales en 1996, seulement 100.000 personnes étaient rentrées dans leur ville d'origine. De surcroît, 80.000 personnes avaient été chassées à nouveau de leurs foyers dans la même période ${ }^{16}$. Cela signifie que, jusqu'en 1996, le nettoyage ethnique continuait sous la forme d'un «nettoyage électoral». Étant donné l'impossibilité d'écarter les anciens combattants de la nouvelle scène politique et la continuation du nettoyage ethnique, la réconciliation après les premières élections se révéla précaire. Tout en se reconnaissant de manière réciproque, les différentes communautés du pays demeuraient cantonnées dans leurs entités territoriales respectives. Bien évidemment, une certaine esquisse de réconciliation politique fondée sur la reconnaissance réciproque résulte aussi bien de la signature de l'accord de Dayton que des premières élections générales. Le maintien du cessez-le-feu, le retour des armées à leurs casernes et la démobilisation des combattants pendant le scrutin en témoignent. Mais la question de la réconciliation morale reste ouverte. Les joutes électorales à l'encontre de la réconciliation morale Mécanisme de participation politique à appliquer dans une société dépourvue d'une véritable unité sociale et politique, les élections constituent un enjeu fondamental de la reconstruction en Bosnie, d'autant plus que l'arrêt des hostilités conclu à Dayton n'est qu'une solution partielle au conflit. En effet, l'accord de Dayton n'accomplit pas tout l'exercice de pacification. Il constitue plutôt une sorte d' « armistice intercommunautaire » dont la préoccupation principale est l'établissement de lignes de partage entre les deux entités politiques de la Bosnie (la Fédération croatobosniaque de Bosnie et Herzégovine et la République serbe de Srpska) ${ }^{17}$. Il s'agit donc d'un cessez-le-feu qui institue une division de facto entre les trois communautés du pays, sans jamais contrebalancer cette division par des mécanismes de cohabitation intercommunautaire. Si la vertu de l'accord de Dayton est celle de l'établissement d'une paix garantie par une force militaire à caractère multinational ('IFOR, devenue peu après la SFOR), la réconciliation y est à peine en phase de gestation. La paix de Dayton n'exprime en effet que l'irréductibilité réciproque des adversaires militaires dans le champ de bataille. Il s'agit donc d'une paix précaire et fragile ${ }^{18}$. Afin d'accomplir la tâche pacificatrice, la cessation d'hostilités requiert l'encadrement de l'inimitié par des institutions et/ou des idéologies qui l'inscrivent dans un intérêt commun ${ }^{19}$. D'où le besoin de mécanismes pour rendre possible le passage de la paix en tant qu'imposition de l'extérieur à la paix en tant qu'aspiration interne. L'intervention de la communauté international après l'accord de Dayton vise justement à établir un ensemble d'initiatives susceptibles de conduire au dépassement des divisions de facto instituées par l'armistice. Un tel projet implique, en principe, un engagement de la communauté internationale dont le but est l'affirmation et la pérennisation de la paix, prévoyant néanmoins à moyen ou long terme le désengagement et le retrait graduels des 
intervenants externes. Au-delà de la reconnaissance réciproque, la réconciliation implique également un processus moral qui suppose la formulation de remords et la demande de pardon : il s'agit bien de devancer le conflit de sorte que la possibilité de renaissance des anciennes hostilités soit éliminée. Bien que la tenue d'élections en Bosnie après la signature de l'accord de Dayton ait contribuée à la reconnaissance de l'ennemi comme interlocuteur légitime, le bilan en termes de réconciliation morale se révèle davantage problématique. Malgré des élections sans incidents majeurs, l'existence formelle de nombreux partis politiques $^{20}$ et une participation élevée ${ }^{21}$, l'exercice électoral ne semble pas renverser les fondements de l'armistice intercommunautaire. Le processus de réconciliation demeure ainsi embryonnaire, et l'élection s'y oppose autant qu'il la favorise. L'atmosphère de haine et d'affrontement qui a souvent entouré les campagnes électorales en Bosnie en est révélatrice. Tout d'abord, la réalisation des élections n'a pas atténué d'une façon significative le discours nationaliste des anciens ennemis. Fondé sur l'idée des clivages communautaires et sur l'impossibilité de cohabitation dans le cadre d'institutions communes fortes, ce type de discours monopolise souvent la grammaire des partis politiques présents sur la scène électorale. Peu de discours pluralistes (appel à l'intégration intercommunautaire) y sont entendus (c.f. Annexe 3). En revanche, de véritables "discours guerriers" rejoignent d'ordinaire les discours nationalistes des partis politiques les plus puissants et les mieux organisés de la scène politique (le SDA bosniaque - devenu KCD en 1998 le HDZ croate, le SDS serbe). Ainsi, le SDA, parti hétérogène qui utilisait un discours plutôt pluraliste avant la signature de l'accord de Dayton, est devenu pendant le scrutin une organisation plus homogène autour d'une aile dure et nationaliste. Lors des élections nationales générales de 1996, par exemple, les leaders d'autres partis bosniaques favorables à la cohabitation avec les autres communautés du pays ont été qualifiés de "traitres » à la cause bosniaque par les membres du SDA. Pendant la campagne électorale de 1996, certains membres du SDA ont même fait appel au « retour et à la reconquête militaire » de certaines villes occupées par les Serbes, comme celle de Banja Luka ${ }^{22}$. Les discours des partis les plus importants des autres communautés de la Bosnie ne sont guère différents de celui du SDA. En 1996, un clip de propagande du SDS serbe a été diffusé à la télévision de Pale, comportant des images de cavaliers serbes du XIVème siècle aux prises avec des soldats turcs et ayant pour message initial: "Des siècles d'esclavage prennent fin, les Serbes se lèvent et brandissent leur drapeau ». Le clip finissait avec un dernier slogan : « Un Serbe ne se mettra plus jamais à genoux ». De la même façon, les leaders du HDZ croate ont évoqué dans leurs meetings électoraux «la force du peuple croate » et l'existence même après la guerre de leur « république autoproclamée " de Herzeg-Bosna ${ }^{23}$. Cette orientation guerrière des discours nationalistes montre bien les difficultés de la réconciliation morale. Bien évidemment, cette joute de discours guerriers a contribué à entretenir des dynamiques de nonconciliation et de non-rapprochement communautaire, et même d'affrontement. Il s'agit bien de logiques belliqueuses qui imprègnent la scène politique locale. Ces logiques tendent à se pérenniser malgré la signature d'un accord de paix, non qu'elles soient imposées par les anciens ennemis dans la joute électorale, mais parce qu'elles sont bien accueillies par un électorat frappé encore par la peur. En effet, quelques mois après la fin de la guerre, l'électorat est encore terrorisé. Bien qu'une certaine réconciliation vis-à-vis de l'ennemi public s'opère à travers le cessez-le-feu, l'inimitié privée continue à nourrir les peurs de la société. L'électorat des sociétés qui sortent d'une situation de conflit est souvent à la recherche de la protection plutôt que de la 
réconciliation. Dans ce contexte, les partis nationalistes réussissent facilement à se projeter comme des remparts contre une agression possible de l'ennemi. D'autant plus qu'ayant le contrôle des armées et des polices de chaque communauté, ils fournissent une certaine fonction de protection effective. Par exemple, en mai 1996, après la première réunion en public du SDA, la première parade militaire du gouvernement d'Alija Izetbegovic a eu lieu dans la Fédération croato-bosniaque. La stratégie du SDA, alors au pouvoir, était évidente: il s'agissait de montrer que le parti avait doté le peuple musulman d'une capacité de défense vis-à-vis des autres communautés de la Bosnie $^{24}$. Sans doute, le climat de terreur qui frappe l'électorat est-il avant tout le produit du souvenir de la guerre. Pourrait-on envisager l'effacement d'un tel climat au fur et à mesure que le temps rend possible l'oubli ? La tenue d'élections semble s'y ériger en obstacle : en mettant sur scène les discours nationalistes, elle rafraîchit la mémoire. Les effets se succèdent ainsi comme un cercle vicieux dans lequel le climat de guerre latente renforce les options nationalistes et les discours de revanches guerrières. Les résultats électoraux jouent dans cette dynamique circulaire un rôle non-négligeable: ils témoignent de l'efficacité du discours nationaliste dans la mobilisation d'un électorat et la consolidation d'une force électorale ${ }^{25}$. De fait, les trois partis qui refusent l'idée d'une réintégration intercommunautaire au-delà de l'unité politique juridique et formelle proposée par l'accord de Dayton (le SDA bosniaque devenu KZD pour les élections de 1998 - le HDZ croate et le SDS serbe) sont devenus les principaux détenteurs du pouvoir en Bosnie. En comparant les élections de 1996 à celles de 1998, il est aisé de constater le maintien et l'emprise de ces trois partis à presque tous les niveaux du pouvoir (c.f. Annexe 2). Les résultats des élections donnent ainsi aux acteurs politiques en compétition une image de rentabilité politique du discours nationaliste qui renforce les logiques de non-conciliation de l'armistice intercommunautaire. Bien sûr, le monopole sur la scène électorale des partis nationalistes n'est ni total ni définitif dans le cas de Bosnie. En fait, un grand nombre de partis politiques ont participé aux élections en Bosnie (c.f. Annexe 3), certains espaces s'ouvrant même dans la vie politique à des options plus modérées (la plus remarquable est celle de la coalition serbe modérée Sloga de la République serbe de Srpska, où le SDS serbe a reculé $)^{26}$. Certaines remarques concernant ces espaces et ces options sont cependant nécessaires. Tout d'abord, l'émergence des options modérées s'accompagne de l'affirmation du discours ultra nationaliste et de l'affaiblissement des options pluralistes déjà faibles : ce que l'on peut constater dans les résultats des élections de 1998 (c.f. Annexe 4). Ainsi, malgré la victoire de la coalition Sloga pour la représentation serbe à la présidence nationale collective de la Bosnie, Biljana Plavsic une des têtes de la coalition de modérés - a perdu la présidence de la République serbe de Srpska face au candidat le plus nationaliste : Nicola Poplasen, du Parti radical serbe (SRS). De même, le recul du SDS à l'Assemblée nationale serbe n'a pas seulement favorisé les modérés, mais aussi les ultra-nationalistes du SRS. Le SRS de la République serbe de Srpska s'est déclaré frère du SRS de Serbie, parti dirigé avec beaucoup de succès par un ancien paramilitaire serbe, Vojislav Seselj. Les deux partis défendent encore l'idée d'une union de tous les Serbes dans une seule «Grande Serbie ${ }^{27}$. Par ailleurs, il faut bien discerner les options modérées des pluralistes. Aucun des partis modérés ne prétend le renforcement des attributions des institutions centrales de la Bosnie ; ils n'envisagent que « la bonne cohabitation » entre les communautés dans «le cadre d'entités politiques séparées». Ce sont donc des partis politiques favorables à l'application de l'accord de Dayton, mais pas à son dépassement. Ils n'osent pas 
proposer dans leurs discours politique la réconciliation intercommunautaire. Le multipartisme ne conduit pas donc nécessairement à une offre politique variée. Bien au contraire, le discours nationaliste non-intégrationniste et non-conciliant, qui prétend ériger les partis politiques en boucliers protecteurs des populations, exerce en Bosnie un quasi-monopole (c.f. Annexe 3 et 4). Alors que le contenu du programme est pratiquement le même d'un parti communautaire à l'autre, ce qui change, en revanche, dans les programmes des différents partis politiques, c'est la communauté ciblée. On est donc confronté à une situation de "concentration de l'offre d'idées", pour reprendre la terminologie de Jack Snyder et de Karen Ballentine ${ }^{28}$. Donc, malgré l'existence de certaines alternatives politiques, la tendance à «la concentration de l'offre d'idées » demeure forte en Bosnie. Les alternatives pluralistes y ont tendance à rester à l'écart de la scène politique: soit parce qu'elles représentent un éventail hétérogène de groupes petits et sans aucune expérience politique, qui n'arrivent pas à s'organiser en coalition d'opposition face aux partis nationalistes majeurs; soit parce que les partis nationalistes dominants et au pouvoir ont utilisé plusieurs tactiques de harcèlement et d'isolement des discours pluralistes ${ }^{29}$. C'est bien le cas de le $\mathrm{SBiH}$, dont le leader était Haris Silajdzic, ancien premier ministre du gouvernement d'Alija Izetbegovic et dissident du SDA bosniaque. En tant qu'option pluraliste (son programme promouvait la création d'une Bosnie laïque et unie), le SBiH suscita beaucoup d'expectatives dans les élections nationales de 1996. Au début, la popularité de Silajdzic était forte. Selon un sondage de l'hebdomadaire Dani publié en mai 1996, le SBiH recueillait $42 \%$ des intentions de vote, contre $27 \%$ pour le SDA. Néanmoins, les résultats des élections attribuèrent $80 \%$ des voix au SDA et seulement $14 \%$ au $\mathrm{SBiH}$. Victime de nombreuses tactiques de harcèlement dont l'agression à coups de barre de fer de Silajdzic (juin 1996) ne représenta qu'un des événements, le SBiH fut ainsi progressivement mis à l'écart. On voit bien que les élections tenues après l'accord de Dayton sont loin encore d'éliminer la confrontation physique et d'établir seulement la confrontation verbale. Aucun processus de réconciliation profonde ne peut être envisagé dans un tel contexte de violence. Les électeurs reconnaissent bien cette " concentration de l'offre d'idées". Pour certains, notamment ceux qui habitent à l'extérieur, les options et les alternatives lors de la tenue des élections sont restées très limitées. Les élections, affirment-ils, représentent un choix entre "la peste et le choléra ». En ce sens, les taux élevés de participation, calculés sur le nombre d'électeurs inscrits, ne sont pas indicatifs d'une mobilisation électorale forte autour d'un éventail large d'alternatives. En fait, face aux 3.5 millions d'électeurs inscrits pour les élections de 1990 - les dernières avant la guerre - le nombre d'inscrits est descendu à 2,9 millions en 1996. Si, sur une population totale de 4.365 .000 personnes en 1991, on estime qu'environ 300.000 personnes sont mortes pendant la guerre, cela signifie qu'à peu près autant de personnes n'ont pas voulu s'inscrire en 1996. De plus, il faudrait rendre compte des ressortissants de la Bosnie à l'étranger qui ne se sont pas inscrits. Sur un total de 1.331.833 réfugiés externes en 1996, seulement 641.010 personnes se sont inscrites pour voter depuis l'extérieur, dont 357.193 étaient installées en Croatie et dans la nouvelle Yougoslavie. Plus de personnes auraient pu s'inscrire à l'étranger, notamment dans certains pays d'Europe occidentale, mais elles ne l'ont pas fait (c.f. Annexe 5$)^{30}$. L'absence de véritables alternatives politiques et la peur de légitimer tant les acteurs de la guerre que l'armistice identitaire, semble avoir beaucoup découragé certains réfugiés de voter. L'offre d'idées demeurant étroite, la création d'un processus de dialogue "non-contraint» habermasien en tant que fondement du processus de 
pacification est également difficile. D'autant plus que, toujours selon la terminologie de Snyder et de Ballentine, à "la concentration de l'offre d'idées " vient s'ajouter une "segmentation de la demande d'idées ». Une telle segmentation était assurée dès la signature de l'accord de Dayton car le système électoral établi est fondé sur une base communautaire et identitaire. En consultant le diagramme de l'Annexe 1 de cet article, il apparaît que chaque communauté avait déjà un quota garanti dans la plupart des pouvoirs collégiaux, à savoir, dans la Présidence nationale, les deux Chambres nationales et la Chambre du peuple de la Fédération croato-bosniaque. En prédéterminant le nombre de sièges de chaque communauté mais aussi en séparant les électeurs, le système électoral établi à Dayton n'a pas favorisé l'essor de programmes politiques adressés à différents segments du "marché d'idées", c'est-à-dire, de programmes politiques adressés à toutes les communautés (discours pluraliste nonnationaliste). Il n'a pas non plus favorisé la demande d'idées variées parmi les électeurs, compte tenu du fait que la gamme restreinte des discours empêche la sophistication de la demande. Cette « segmentation de la demande des idées " s'est maintenue lors de la campagne électorale et du scrutin, les idées ne circulant pas facilement entre les communautés. Trois facteurs ont contribué à la circulation restreinte des idées. Premièrement, les médias sont restés cantonnés dans leurs entités d'origine et les journaux n'ont pas été distribués au niveau national ${ }^{31}$. Deuxièmement, presque aucun parti politique n'a mené de campagne électorale à vocation transnationale (c.f. Annexe 3). Autrement dit, le champ de diffusion des idées de presque tous les partis se limite aux frontières des entités politiques établies par l'accord de Dayton. La mobilisation des porteurs d'idées ne se fait pas facilement, soit parce qu'ils n'ont pas toujours les ressources pour le faire, soit parce que les polices des autres communautés posent des obstacles à une telle mobilisation. Troisièmement, vu toutes les difficultés du retour des réfugiés et des déplacés internes à leurs villes d'origine, l'électorat demeure cantonné et fragmenté à l'intérieur d'entités territoriales «ethniquement » homogènes. Bien qu'il ne soit pas possible pour le moment de savoir combien d'électeurs se sont déplacés parmi les deux entités politiques de la Bosnie en septembre 1998, lors des deuxièmes élections nationales générales, la libre mobilité des déplacés n'est pas encore assurée. En effet, l'OSCE a approuvé l'établissement de bureaux de vote pour les réfugiés hors de certaines villes de l'entité politique serbe (Srebrenica et Bratunac, par exemple) où les Bosniaques pouvaient par hypothèse arriver le jour des élections. Selon l'OSCE et l'UNHCR, dans ces villes, vidées de leurs anciens résidents et désormais peuplées par de nouveaux, la paix n'est possible qu'en évitant le contact direct des communautés ${ }^{32}$. Le déplacement libre de la population pendant la réalisation de l'exercice électoral implique des risques non négligeables. Au moins 70\% des réfugiés rapatriés en 1997 et en 1998 ne sont pas encore rentrés dans leurs anciens foyers. Dans la plupart de cas, ils ont été replacés en tenant compte de l'ethnie majoritaire de chacune des zones d'accueil afin d'éviter toute rupture de l'homogénéité ethnique. À plusieurs reprises, l'UNHCR a affirmé que le retour de tous les réfugiés dans leurs foyers d'origine était tout simplement impossible. Bien sûr, l'emprise des leaders nationalistes est facilement assurée à mesure que les populations déplacées par la guerre sont replacées dans des zones relativement homogènes du point de vue « ethnique ». Un tel contexte rend tout modèle dialogique ou délibératif de la réconciliation pratiquement irréalisable. En tant qu'occasion pour le débat public d'idées, les élections peuvent être envisagées comme une pratique de participation politique ayant une fonction intégrative. Dans ce sens et d'un point de vue théorique, les élections pourraient accomplir une fonction de 
réconciliation non négligeable dans les sociétés qui cherchent à sortir d'un conflit. Or, en analysant le passage du communisme vers la démocratie en Yougoslavie, Jack Snyder et Karen Ballentine ont remarqué que, faute de certaines conditions pour encourager le débat public (offre d'idées non-concentrée, demande d'idées nonsegmentée et organes de régulation des élections efficaces), la tenue d'élections déclenche des dynamiques de désintégration, tout en rendant possible le passage de la paix à la guerre ${ }^{33}$. Selon l'analyse présentée ci-dessus, il semble de même que, en l'absence de ces conditions, les élections entravent le processus de réconciliation dans de telles sociétés. Notamment dans sa dimension proprement morale, la réconciliation se heurte aux discours sectaires, voire guerriers, des partis nationalistes, qui sont caractéristiques des sociétés déchirées par la guerre civile. Bien évidemment, la mise en place de toutes les conditions pour la constitution d'un véritable «marché des idées » lors de la tenue d'élections dans les opérations internationales de pacification requerrait un recul temporel significatif vis-à-vis de la cessation des hostilités. Pourtant, l'action de repousser les élections est en même temps difficile et problématique. Tout d'abord difficile, étant donné que la célébration des élections dans le cadre des opérations internationales de pacification engage de multiples acteurs externes. La logique de la responsabilité internationale ne l'emporte pas toujours sur les considérations particulières dans cet ensemble hétérogène d'acteurs, à morphologie variable, dont l'éventail d'intérêts et d'enjeux est large et complexe. L'action de cet ensemble - que l'on appelle "la communauté internationale» - se fonde souvent sur des raisons de politique interne qui ne tiennent compte que marginalement des enjeux du processus de réconciliation. Par exemple, pouvant devenir une affaire délicate à la veille de la campagne présidentielle américaine, les élections en Bosnie de 1996 ne furent pas ajournées malgré l'absence des conditions minimales dénoncée par certains acteurs externes, notamment les ONG et le président de l'OSCE. Afin d'éviter toute critique de la participation des troupes américaines au sein de l'IFOR lors du débat électoral américain, le respect du calendrier établi à Dayton est devenu un impératif pour le Président des États-Unis. Les considérations internes de la puissance américaine ont primé sur l'intérêt général d'améliorer les conditions minimales pour l'organisation du scrutin en Bosnie. L'ajournement des élections est également problématique en ce qu'elle retarde la création d'institutions capables de combler partiellement le vide de pouvoir qui caractérise la sortie d'un conflit. On l'a déjà évoqué : les élections ont une fonction de création de pouvoir ${ }^{34}$. Tel a été l'argument de Carl Bildt, représentant civil de la communauté internationale en Bosnie, lors des discussions sur la pertinence de différer la date de la célébration des élections générales de 1996. D’après lui, les élections auraient au moins permis la mise en œuvre d'une présidence commune à trois têtes et d'un conseil de ministres pluriethnique, ce qui aurait rassemblé autour de la même table les acteurs de la guerre, tout en permettant le dialogue inter-entités et l'esquisse d'une réconciliation politique ${ }^{35}$. Vis-à-vis de ce besoin d'institutionnalisation du politique, la suspension du processus de réconciliation morale est inévitable. La communauté internationale est donc confrontée à un véritable dilemme dans les opérations de pacification internationale: soit elle repousse les élections afin d'améliorer les conditions du débat électoral, en l'absence par définition de pouvoirs ou d'acteurs politiques reconnus et légitimes; soit elle organise le scrutin rapidement, suspendant la question morale de la réconciliation. Parce que la pacification politique semble un préalable à toute réconciliation morale, l'ajournement des élections s'avère impossible. D'autant plus que la communauté internationale ne semble pas avoir à sa 
disposition un meilleur mécanisme d'institutionnalisation du politique : les élections constituent non seulement la «forme minimale » de participation politique, mais aussi celle qui concerne le plus grand nombre d'individus. Il ne reste ainsi qu'à envisager l'approfondissement du processus de réconciliation grâce à la prolongation de l'action d'ingérence de la communauté internationale. En effet, dans le cas de la Bosnie, la communauté internationale a essayé a posteriori de renverser les résultats électoraux non désirés, en réalisant de nouvelles ingérences à plusieurs reprises. Par exemple, lors des élections nationales de 1998, la communauté internationale a voulu favoriser les modérés serbes, en versant des aides financières massives pour la reconstruction des régions serbes sous leur contrôle. La stratégie des exécutants de l'exercice de pacification était claire: il s'agissait de convaincre la population que l'avenir économique appartenait aux réconciliateurs. De même, lors de l'élection du président de l'Assemblée nationale de l'entité politique serbe en novembre 1998, le haut représentant civil de la communauté internationale pour la Bosnie, Carlos Westendorp, n'a pas hésité à intervenir et à promouvoir des accords et des marchandages entre les Serbes modérés et les Bosniaques de l'entité politique serbe, afin d'obtenir pour les modérés la présidence du corps législatif ${ }^{36}$. Il ne faut pourtant pas négliger les capacités d'adaptation des acteurs locaux aux interventions externes. Ces acteurs peuvent toujours renverser les ingérences en leur faveur, tout en allant à l'encontre de l'exercice de pacification. Il s'agit bien d'une série d' « effets pervers » de l'ingérence. Par exemple, l'ingérence en Bosnie a provoqué des réactions et des contestations fortes de la part des nationalistes et des ultra nationalistes. En considérant que la Bosnie est devenue une sorte de "protectorat international » dont le gouverneur serait le haut représentant civil de la communauté internationale, ces deux courants politiques sont arrivés à séduire une bonne partie de l'électorat de l'entité politique serbe ${ }^{37}$. Ainsi, sans le vouloir, l'ingérence peut renforcer les acteurs et les partis politiques les plus opposés à tout processus de réconciliation ${ }^{38}$. Somme toute, les élections ne constituent pas en soi un instrument de réconciliation. Bien que la «mythologie démocratique » tende à présenter le vote comme un choix rationnel et individuel, hors de contraintes et de déterminants externes, les élections en Bosnie montrent bien à quel point les élections sont une pratique de participation politique conditionnée. Les conditions du déroulement du processus électoral s'avèrent donc plus importantes pour le processus de réconciliation que le vote en soi : de telles conditions déterminent non seulement l'éventail de choix mis à la disposition de l'électorat (très réduit dans le cas de la Bosnie), mais aussi la possibilité d'affirmer une culture politique et une dynamique de la politique davantage fondées sur une logique de réconciliation. Nécessaires à tout processus de pacification politique, les élections se révèlent en revanche insuffisantes à tout processus de réconciliation morale.

\section{NOTES}

1. Lagroye J., Sociologie politique. Troisième édition. Presses de Sciences Po et Dalloz, Paris, 1997, p. 330. 
2. Ibid., pp.287-290 et 332.

3. Introduction de l'Annexe III de l'accord de Dayton. http://www.ohr.int/gfa/gfaan3.htm.

4. Les remarques et les conclusions présentées ici proviennent de l'observation de cinq scrutins qui ont eu lieu en Bosnie depuis la signature de l'accord de Dayton : les élections de la ville de Mostar (juin 1996), les deux élections nationales (l'une en septembre 1996, l'autre en septembre 1998), les premières élections municipales (septembre 1997), et les élections extraordinaires législatives de l'entité politique serbe (novembre 1997). Les élections de Mostar, « laboratoire » des élections postérieures, appelèrent les électeurs à choisir le maire, le conseil général et les conseils des six districts de la ville. Dans les élections nationales furent élus les membres de la Présidence nationale collective tripartite, de la Chambre de représentants de l'Assemblée parlementaire nationale, des corps législatifs des deux entités politiques de la Bosnie (la Chambre fédérale de représentants et la Chambre du peuple dans la Fédération croato-bosniaque de Bosnie et Herzégovine ; et l'Assemblée nationale dans la République serbe de Srpska), des assemblées cantonales de l'entité politique croatobosniaque, des assemblées municipales, ainsi que le Président de l'entité politique serbe. Les premières élections municipales dans les deux entités politiques de la Bosnie devaient avoir lieu en même temps que les premières élections nationales, mais furent reportées jusqu'à septembre 1997, à cause de multiples irrégularités. Enfin, les électeurs de l'entité politique serbe (la République serbe de Srpska) furent appelés aux urnes par leur président, Biljana Plavsic, pour des élections législatives hors du calendrier électoral établi par l'accord de Dayton. Ces élections eurent pour but le dépassement de la crise gouvernementale interne déclenchée par les forces ultranationalistes siégeant à l'Assemblée nationale de cette république. L'Annexe 1 de cet article présente un diagramme de l'architecture institutionnelle et du système électoral mis en place en Bosnie par l'accord de Dayton. Les résultats des élections nationales sont présentés dans l'Annexe 2.

5. L'accord de Dayton ne définit clairement ni la nature ni le cadre institutionnel d'une véritable unité politique. La nature de l'État bosnien demeure indéfinie dans l'accord de Dayton, la formule d' « un seul État constitué par deux entités politiques » étant à la fois vague et ambiguë. Le cadre institutionnel de cohabitation apparaît également bien flou, puisque les pouvoirs des institutions communes aux deux entités politiques de la Bosnie (la Présidence collective, l'Assemblée parlementaire nationale et la Cour constitutionnelle) sont minimaux. Ce gouvernement central ne détient que certaines attributions limitées en matière de politique étrangère, de commerce international, de politique monétaire, de communication, de mise en œuvre des lois internationales, de migration et de contrôle des frontières. Pouvant être facilement bloquées par les initiatives des deux entités politiques de la Bosnie, les institutions centrales sont dépourvues d'un pouvoir réel. D'autant plus qu'en gardant leurs propres parlement, gouvernement, forces de police et armée, chacune de ces deux entités détient en fait la majorité des fonctions propres d'un État. Sur le caractère fictif de l'unité politique créée à Dayton : Sorel J.M., « L'accord de paix sur la Bosnie-Herzégovine du 14 décembre 1995 : un traité sous bénéfice d'inventaire ", in Annuaire français de droit international, XLI, 1995, pp.64-99 ; Fournier J., « La crise yougoslave : la genèse du conflit et ses perspectives de paix dans l'après-Dayton », in Revue Études Internationales, Vol.XXVIII, n³ 3, septembre 1997, pp.461-491. 
6. D'après l'Annexe III de l'accord de Dayton les conditions à remplir pour la tenue d'élections libres et démocratiques sont les suivantes : l'établissement d'un environnement politique « neutre »; la protection du droit de vote secret ; l'absence de campagnes d'intimidation ou de terreur ; la liberté d'expression et de la presse ; la liberté d'association, notamment en ce qui concerne la constitution de partis politiques ; la liberté de mouvement des populations ; le respect des droits de l'homme reconnus au niveau international.

7. Pour l'accomplissement de toutes les tâches de régulation et d'organisation des élections, l'accord de Dayton prévoit la constitution d'une Commission provisionnelle dans laquelle les parties en conflit doivent être représentées à côté des représentants de la communauté internationale. Néanmoins, compte tenu du fait que l'accord de Dayton établit le primat de l'opinion du chef de la mission de l'OSCE dès l'instant où les membres de cette Commission sont en désaccord, celui-ci, dans la pratique, a assumé seul les attributions de régulation des comices électoraux. Voir les articles 2 et 3 de l'Annexe III (accord de Dayton).

8. Les premières élections nationales devaient avoir lieu dans les six premiers mois suivant l'entrée en vigueur de l'accord de paix, avec un délai maximum de neuf mois si l'OSCE l'estimait nécessaire. Voir : article 2 de l'Annexe III (accord de Dayton).

9. Pour les élections nationales de 1996, il y avait en Bosnie 55000 soldats de l'IFOR et 900 observateurs internationaux. Pour celles de 1998, 32500 soldats de la SFOR de l'OTAN, 2000 policiers internationaux et 3200 observateurs électoraux.

10. Voir : article 4 de l'annexe III (accord de Dayton).

11. Schmeets H., Exel J., The 1996 Bosnia-Herzegovina Elections. An analysis of the observations, Dordrecht/Boston/Londres, Kluwer Academic Publishers, 1997.

12. Igric G., « Profiteurs de guerre », Le Monde diplomatique, juin 1996.

13. « Bosnie : les municipales reportées », Le Figaro, 28 août, 1996. « What kind of Bosnia?", The Economist, 24 august 1996.

14. Defouloy E., "Les réfugiés bosniaques commencent à voter ", Le Monde, 30 août 1996.

15. La plupart de ces déplacements eurent lieu grâce à une vingtaine de routes de trajets d'autobus mis en place par l'OSCE sous la protection d'une quantité considérable de soldats de l'IFOR. Schmeets et Exel, op.cit., p.104. De la Gorce P. M., « Amers lendemains d'élections en Bosnie », Le Monde diplomatique, novembre 1996. 16. Ourdan R., « Elections municipales en Bosnie », Le Monde, 16 août 1996. 17. Si l'on regarde de près l'accord de Dayton, la plupart des dispositions se référent à ce que l'on appelle l'« armistice intercommunautaire » : les annexes 1A (les aspects militaires de l'accord), 1B (la stabilisation et la limitation de l'armement), 2 (la frontière entre les deux entités) et 11 (la police internationale). Certes, il y a d'autres dispositions où la reconstruction de l'État et le dépassement de l'armistice semblent centrales : les annexes 3 (les élections), 4 (la constitution de la Bosnie-Herzégovine), 6 (les droits de l'homme), 7 (les réfugiés), 8 et 9 (la restauration de monuments nationaux et la création des entreprises publiques nationales), 10 (les aspects civils du plan de paix). Ces dispositions sont pourtant assez vagues, voire parfois contradictoires. Elles demeurent ainsi des dispositions secondaires de l'accord de Dayton.

18. L'armistice de Dayton est avant tout un accord " minimum de cohabitation », signé dans une conjoncture particulière, dans laquelle les parties belligérantes ont été sensibilisées, d'une part, aux récompenses promises par un accord de paix dessiné par un médiateur manipulateur et musclé (les États-Unis) et, d'autre part, aux coûts de la 
poursuite de la guerre - elle aussi nourrie par l'intervention de ce médiateur. Le consentement des belligérants à la signature de l'armistice est ainsi la résultante d'une évaluation en termes de coûts et de bénéfices immédiats, de toute façon instables à long terme.

19. En réfléchissant sur la pacification, il est probablement plus correct de parler d'« encadrement » de l'inimitié que de « dépassement ». Notamment, si l'on tient compte de l'ambiguité du terme " inimitié », soulignée par Schmitt. D'après cet auteur, dans la guerre inter-étatique, l'ennemi est un véritable ennemi : il peut être diabolisé par des discours nationalistes hystériques. Mais dès lors qu'un certain nombre de règles de conduite sont inscrites dans l'ethos professionnel des militaires et des diplomates, l'inimitié demeure compatible avec l'idée d'une paix honorable. Sans doute, ces règles de conduite sont bien des institutions qui font barrage aux débordements indéfinis de l'inimitié inter-étatique. De même, bien que sur un registre très différent, il nous semble que l'inimitié intra-étatique peut être encadrée par des institutions et/ou des idéologies qui l'inscrivent dans un intérêt commun. Dans ce sens, il est possible de penser à certaines formes institutionnelles plus solides que d'autres devant l'inimitié. À propos de la lecture "schmittienne » de l'inimitié, voir le texte introductif de John Crowley dans ce numéro de Culture \& Conflits.

20. On constate un nombre élevé de partis politiques et de candidats inscrits auprès de l'OSCE pour les deux élections nationales générales de la Bosnie. En septembre 1996, par exemple, l'OSCE a enregistré 49 partis politiques, dont 13 pour la Présidence collective, 20 pour la Chambre des représentants de la Bosnie, 13 pour la Chambre des représentants de la Fédération croate-musulmane, 16 pour l'Assemblée nationale de la République serbe. Udovicic R., "Elections in Bosnia : Standing in place », in Uncaptive mind, vol.9, n 1-2, winter-summer, 1996-1997. En septembre 1998, 83 partis politiques, coalitions et candidats indépendants se sont inscrits aux élections. Cf. OSCE Web Site. 21. Le taux de participation aux élections nationales générales de septembre 1996 fut proche de $70 \%$ et celui des élections municipales de septembre 1997 de $85 \%$.

22. Ourdan R., « Le Parti nationaliste d'Alija Izetbegovic mène une campagne électorale agressive en Bosnie », Le Monde, 4 septembre 1996.

23. Ourdan R., « Les élections générales en Bosnie vont renforcer le pouvoir des nationalistes ", Le Monde, 14 septembre 1996.

24. «La Bosnie vaut-elle vraiment une messe électorale ? ", Courrier international, 20 juin 1996.

25. UDOVICIC, op.cit.

26. Lors des élections législatives de novembre 1997, convoqués par Baljina Plavsic pour surmonter l'opposition des ultra-nationalistes du Parti démocratique serbe (SDS), les modérés de l'Alliance populaire serbe (SNS) augmentèrent leur nombre de sièges au sein de l'Assemblée nationale de leur entité. Lors des deuxièmes élections générales nationales de septembre 1997, presque le même groupe de modérés a formé une coalition (Sloga), dont le candidat (Zivco Radisic) a remporté le siège serbe de la Présidence collective de Bosnie. En août 1998, cette coalition a remporté aussi la présidence de l'Asemblée nationale de l'entité politique serbe.

27. En témoignent, les menaces d'expulsion des postes de travail des électeurs déloyaux ou traîtres, le sabotage de meetings en mobilisant des mères vêtues de noir ayant perdu leurs enfants pendant la guerre, les saisies d'affiches et l'expulsion des villes des candidats d'opposition, l'exercice de la violence physique et les menaces de mort contre 
ces candidats, l'interdiction d'accès des idées alternatives aux médias contrôlés par les partis nationalistes. "Serbs law », Financial Times, 19 September 1998.

28. D'après Snyder et Ballentine, la concentration de l'offre d'idées dans une joute électorale ne se mesure pas à partir du nombre de partis politiques sur place, mais sur le spectre large ou étroit de programmes politiques en concurrence. Snyder J., Ballentine K., « Nationalism and the Marketplace of Ideas », in International Security, vol.21, n² , autumn, 1996.

29. Ourdan R., « Les oppositions tentent de s'unir pour vaincre les nationalistes au pouvoir », Le Monde, 15 mai, 1996 ; Zivanovic M. (entretien), in Uncaptive mind, vol.9, $\mathrm{n}^{\circ}$ 1-2, winter-summer, 1996-1997. Tokic S. (entretien), in Uncaptive mind, vol. 9, n 1-2, winter-summer, 1996-1997.

30. Defouloy, op.cit.

31. Un exemple frappant de la circulation limitée des idées est la ville de Mostar. Pendant les élections de 1996, les journalistes de Mostar-Est, le côté bosniaque, n'ont pas osé s'aventurer à Mostar-Ouest, le côté croate. La ville connaissait en fait à ce moment-là deux journaux différents, chacun d'entre eux contraint de circuler dans l'espace restreint de sa propre communauté. « Les élections générales en BosnieHerzégovine auront lieu samedi 14 septembre », Le Monde, 27 juin, 1996 ; James L., « Media late for Bosnian Vote Date », International Herald Tribune, 16 august 1996. 32. «Bosnie : les réfugiés musulmans de Srebrenica renoncent à voter ", Le Monde, 15 septembre 1998

33. Syder, Ballentine, op.cit.

34. Les sociologues affirment que voter c'est accepter « une règle de jeu qui contribue puissamment à l'établissement d'un ordre politique spécifique ». Lagroye, op.cit.

35. «La Bosnie vaut-elle .... », op.cit.

36. Lecomte C., « Les Serbes modérés de Bosnie emportent la présidence du Parlement », Le Monde, 6 novembre, 1998.

37. « Six scrutins pour un pays divisé », L'Humanité, 14 septembre 1998.

38. « Bosnie : cuisante défaite des modérés », Le Figaro, 22 septembre 1998.

INDEX

Index géographique : Balkans, Bosnie-Herzégovine

Mots-clés : démocratie, maintien de la paix, sortie de conflits, gestion de conflits 